\title{
Primary hyperoxaluria type 1 - two case reports
}

\author{
Inês Ganhão ${ }^{1}$, Catarina Borges ${ }^{2}$, Marta Amorim ${ }^{3}$, Marisa Braga da Cruz ${ }^{3}$, Susana Nobre ${ }^{4}$, Telma Francisco ${ }^{5}$, Dinorah Cardoso ${ }^{6}$, Margarida Abranches $^{5}$ \\ 1 Pediatrics Department, Centro Hospitalar Barreiro-Montijo, Portugal \\ 2 Pediatrics Department, Unidade Local de Saúde do Baixo Alentejo, Portugal \\ ${ }^{3}$ Genetics Department, Centro Hospitalar Universitário de Lisboa Central, Portugal \\ ${ }^{4}$ Pediatric and Adult Liver Transplantation Unit, Centro Hospitalar Universitário de Coimbra, Portugal \\ ${ }^{5}$ Pediatric Nephrology Unit, Centro Hospitalar Universitário de Lisboa Central, Portugal \\ ${ }^{6}$ Pediatric Surgery Department, Centro Hospitalar Universitário de Lisboa Central, Portugal
}

\section{ABSTRACT}

Background: Primary hyperoxaluria type 1 is a rare autosomal recessive inherited disease, caused by mutations in AGXT gene, with an estimated incidence of 1:100.000 live births per year in Europe. Over 50\% present with end stage renal disease at diagnosis. Case reports: The first case is a 14-year-old boy, second child to consanguineous parents, with history of recurrent lithiasis and ureteral dilatation starting 5 years before. Urine/stone analysis revealed calcium oxalate monohydrate crystals and markedly elevated urine oxalate excretion. Genetic tests confirmed a mutation in AGXT gene, c.1151T>C, in homozygosity. Two years after, nephrocalcinosis was identified and glomerular filtration rate gradually declined. Oxalate deposition in solid organs was excluded and successful orthotopic liver transplantation was performed, with stabilization of glomerular filtration rate.-The second case is a 16 -year-old girl, with recurrent episodes of renal colic. At diagnosis, she had obstructive hydronephrosis, multiple kidney stones and an estimated glomerular filtration of $42.1 \mathrm{~mL} / \mathrm{min} / 1.73 \mathrm{~m}^{2}$. Metabolic study showed hypocitraturia and hyperoxaluria. With dietetic measures and irregular treatment, urine oxalate excretion remained high but renal function improved. Genetic tests confirmed the presence of two pathologic variants in AGXT gene: c.731T >C and c.1151T>C in compound heterozygous. Conclusions: Recurrent urolithiasis and nephrocalcinosis in children along with family history/consanguinity should raise the suspicion of Primary Hyperoxaluria type 1. Conservative treatment may increase renal survival. Effects of systemic oxalosis must be screened when glomerular filtration rate declines below $30-50 \mathrm{~mL} / \mathrm{min} / 1.73 \mathrm{~m}^{2}$, and sequential or combined liver and kidney transplantation should be considered.

Keywords: AGXT gene, end stage renal disease, primary hyperoxaluria, renal lithiasis, transplantation

\section{BACKGROUND}

Primary hyperoxaluria type $1(\mathrm{PH} 1)$ is a rare autosomal recessive inherited disease, with an estimated incidence of 1:100 000 live births per year in Europe, more prevalent in countries where consanguineous marriages are common, such as Kuwait, Tunisia and Egypt. ${ }^{1-4}$ It is caused by mutations in AGXT gene, leading to liver-specific peroxisomal enzyme alanine-glyoxylate aminotransferase (AGT) dysfunction. This failure to detoxify glyoxylate results in oxalate overproduction, with excessive urinary excretion of oxalate. Oxalate is poorly soluble and forms calcium oxalate crystals, leading to recurrent urolithiasis and/or nephrocalcinosis, culminating in renal failure. ${ }^{1,2,5,6}$ Over $50 \%$ already present with end stage renal disease (ESRD) at time of diagnosis. ${ }^{1}$ Progressive decline in estimated glomerular filtration rate (eGFR) results in reduced oxalate excretion by the kidneys, while the liver continues to product excess of oxalate, leading to increased plasma oxalate levels (oxalosis) and systemic oxalate deposition in several organs. ${ }^{2,5,6}$

\section{CASE REPORTS}

\section{Case 1}

The first patient, a Caucasian male, was referred to our hospital at the age of 9 for investigation of recurrent lithiasis, with several episodes of stone elimination, starting 6 months before, and ureteral dilatation. He was the second child to consanguineous parents (second degree cousins), with unremarkable past history, except for monosymptomatic primary enuresis and minor beta-thalassemia. Regarding his family medical history, no relevant renal disease history was known, except for an isolated episode of renal colic of his father at the age of 35 . Blood analyses showed an estimated glomerular filtration rate (eGFR) of $32.3 \mathrm{~mL} / \mathrm{min} / 1.73 \mathrm{~m}^{2}$, normal ionogram and no other relevant findings. Renal ultrasonography (US) confirmed bilateral renal lithiasis causing right ureter obstruction and nonspecific parenchymal hyperechogenicity. Due to urinary retention and calculus in the urinary meatus, the stone was surgically removed, without complications. The spectroscopic analysis of the calculus identified calcium oxalate.

24-h urine showed markedly elevated urine oxalate excretion (2.22 $\mathrm{mmol} / 1.73 \mathrm{~m}^{2} /$ day; reference value $<0.5 \mathrm{mmol} / 1.73 \mathrm{~m}^{2} /$ day). Besides hydration, he was treated with Uralyt ${ }^{\circledR}$ (sodium and potassium citrate) and pyridoxine, with clinical and laboratory improvement.

At outpatient clinic appointment, the family was investigated and neither the parents nor the brother had nephrolithiasis or augmented urine oxalate excretion. The eGFR continued to improve until 93.7 $\mathrm{mL} / \mathrm{min} / 1.73 \mathrm{~m}^{2}$ at the age of 10 , with conservative measures. However, despite increasing doses of the medication above, urine oxalate excretion remained more than 3 -fold higher than reference values. 


\section{Figure 1}

Renal ultrasonography showing nephrocalcinosis (case 1).

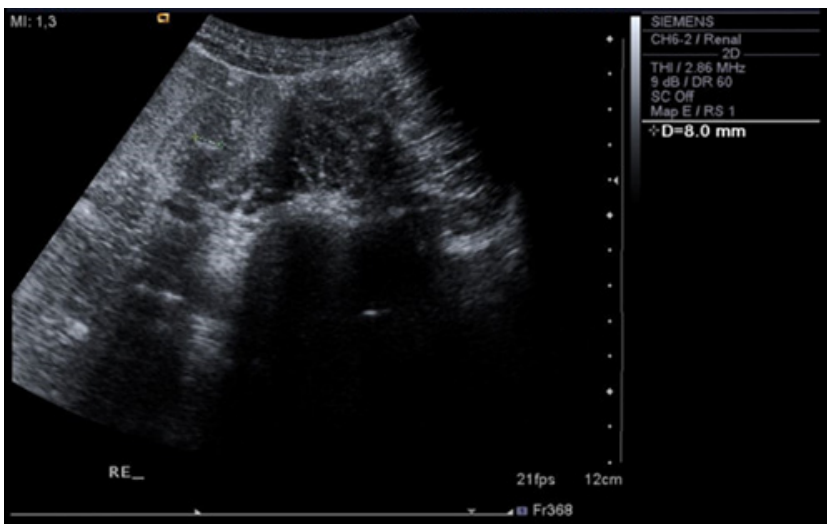

Therefore, he was referred to the Genetics Department and genetic tests confirmed a mutation in AGXT gene, c.1151T>C, in homozygosity. He was under clinical, laboratory and imaging follow-up while receiving conservative treatment. Approximately two years after initial symptoms, nephrocalcinosis was identified in ultrasonography (Figure 1), eGFR gradually declined, and albuminuria and uricemia developed. Systemic oxalate deposition in myocardium, retina, bone marrow and thyroid were excluded following a multidisciplinary approach.

At 14 years old, eGFR decreased below $40 \mathrm{~mL} / \mathrm{min} / 1.73 \mathrm{~m}^{2}$ and he was then proposed for a sequential liver-kidney transplantation. Orthotopic liver transplantation of deceased donor was performed complicated by gastrointestinal bleeding caused by duodenal ulcer at day 15 post-transplantation and stenosis of hepatic artery with need for stent placement seven months later. Kidney transplantation was not performed yet because eGFR pre-transplantation was stable at around $40 \mathrm{~mL} / \mathrm{min} / 1.73 \mathrm{~m}^{2}$ and improved after transplantation until $45.5 \mathrm{~mL} /$ $\mathrm{min} / 1.73 \mathrm{~m}^{2}$. Currently, ten months after liver transplantation, he has normal urine oxalate excretion and hepatic enzymes. He maintains triple immunosuppression, with tacrolimus, prednisolone and mycophenolate mofetil, and double antiaggregation (due to stent placement).

\section{Case 2}

The second patient is a Caucasian 16-year-old girl, with no relevant past personal history or consanguinity. Regarding family history, the patient has a paternal uncle and a $1^{\text {st }}$ degree cousin with renal lithiasis. She initiated recurrent episodes of renal colic at 13 years of age. Approximately 6 months after the initial symptoms, she was admitted to a tertiary hospital. Abdominal radiography and renal ultrasonography revealed multiple bilateral kidney stones, 6-10 $\mathrm{mm}$ of diameter (Figure 2 ), obstructive right hydronephrosis ( $35 \mathrm{~mm}$ of posteroanterior diameter) and ureteral dilation upstream a $12 \mathrm{~mm}$ stone. Serum creatinine was elevated $\left(1.55 \mathrm{mg} / \mathrm{dL}\right.$ ), eGFR $42.1 \mathrm{~mL} / \mathrm{min} / 1.73 \mathrm{~m}^{2}$, with no acidosis or other relevant changes in blood analysis. A ureteral stent was placed, with improvement of hydronephrosis and renal function.

\section{Figure 2}

X-ray showing multiple radiopaque stones (case 2).

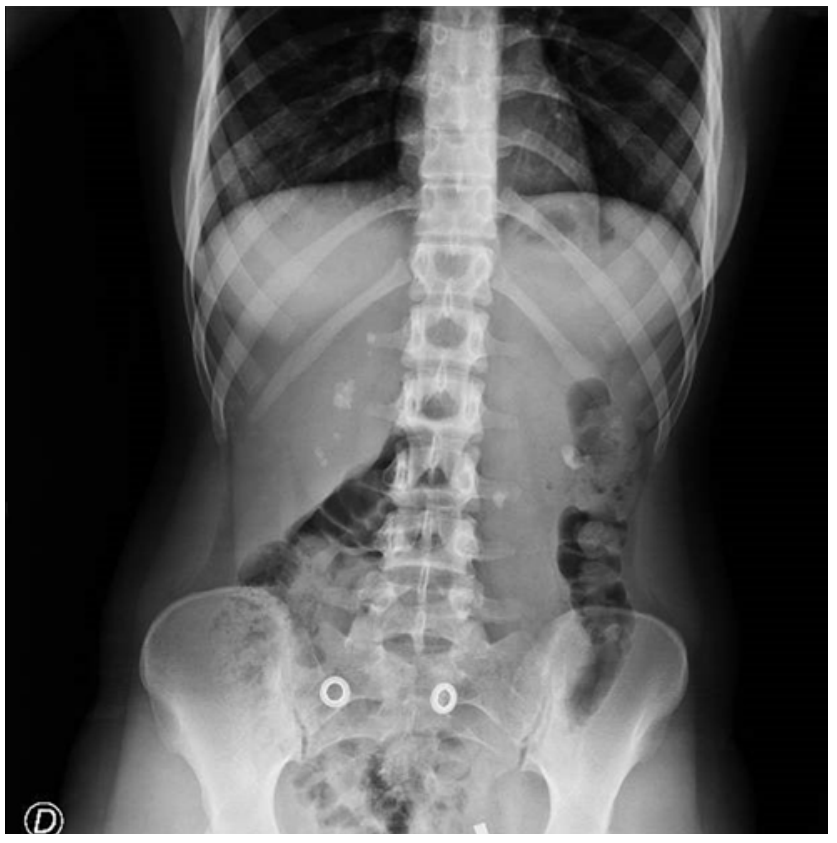

At the outpatient clinic appointment, metabolic study showed hypocitraturia and hyperoxaluria. She was found to have a very high oxalate and low citrate dietary intake. Stone analysis was not possible. Conservative treatment (sodium and potassium citrate and, later, pyridoxine) was prescribed, with irregular compliance. During follow-up, surgery was twice required for stone fragmentation and stent replacement. Despite diet improvement and treatment, urine oxalate excretion remained 2- to 4 -fold higher than reference values. Genetic tests confirmed mutations in AGXT gene, with pathologic variants c.731T >C and c.1151T >C in compound heterozygosity, hence the diagnosis of $\mathrm{PH} 1$.

At the present time, she is 16 years old, maintains recurrent episodes of renal colic and irregular compliance with the treatment. However, urine oxalate excretion has significantly improved and eGFR stabilized at around $66 \mathrm{~mL} / \mathrm{min} / 1.73 \mathrm{~m}^{2}$. Efforts are being made to improve therapeutic compliance, in order to delay disease progression.

\section{DISCUSSION}

Primary Hyperoxaluria type 1 has a wide spectrum of clinical presentation and proves a diagnostic challenge. It may present at any age and clinical manifestations range from infantile massive nephrocalcinosis and failure to thrive to recurrent or only occasional stone formation. ${ }^{1,2,5}$ Recurrent urolithiasis and/or progressive nephrocalcinosis are most often the first symptoms and, particularly if there is a suggestive family history or consanguinity, these should raise the suspicion of $\mathrm{PH} 1$ and motivate a metabolic screening. ${ }^{1,5}$ Some patients 
even remain asymptomatic and ESRD is their first manifestation. ${ }^{2}$ ESRD is present at diagnosis in more than $50 \%$ of the patients. ${ }^{1,5}$ Studies demonstrate an average of 5 years from the first symptoms to the definitive diagnosis that may be attributed to the heterogeneous presentation and limited knowledge of the disease among medical community. ${ }^{5}$ In both cases discussed above, one and a half years passed between initial symptoms and suspicion of diagnosis and another one and a half years until definitive diagnosis. Clinicians differentiate 5 major presentations: 1 ) infantile form (26\%), with early nephrocalcinosis and kidney failure; 2) childhood or adolescent form (30\%) with recurrent urolithiasis and progressive renal failure; 3 ) adult form (30\%) with occasional renal stone formation; 4) post-kidney transplantation form (10\%); and 5) pre-symptomatic form (13\%), with family history. ${ }^{5}$ Beyond this clinical variability, there is also a genetic heterogeneity; more than 178 pathological mutations have been described on the AGXT gene. ${ }^{7}$ Definitive diagnosis is based on mutation analysis. ${ }^{1}$ Genetic testing is also useful for family counselling (establishing the recurrence risk and offering prenatal and preconceptional options), especially in places where $\mathrm{PH} 1$ is prevalent.

Conservative treatment (to decrease oxalate production and increase calcium-oxalate solubility) may increase renal survival; therefore it should be started when the disease is first suspected. ${ }^{1,5}$ In case 1 , conservative treatment proved helpful in the first five years and, in case 2 , efforts are being made to improve compliance.

While age of onset and severity of disease is variable and not necessarily predictable by genotype, a correlation between pyridoxine responsiveness and homozygosity for the p.Gly170Arg mutation has been observed. Patients with Gly170Arg mutation respond to treatment with vitamin B6 (pyridoxine), while others do not. This is valuable because not all patients have been shown to be responsive to pyridoxine, and strategies that help to identify the individuals most likely to benefit from such targeted therapies are desirable. ${ }^{8}$

When eGFR falls below $30-50 \mathrm{~mL} / \mathrm{min} / 1.73 \mathrm{~m}^{2}$, the systemic effects of oxalosis must be screened. Systemic deposition of oxalate may occur in bone marrow (the major accumulation site), heart, retina, skin, blood vessels, and nervous system, among others. ${ }^{2,5}$ The second patient still awaits screening because diagnosis has only been made recently and eGFR is steady.

In patients where pre-emptive liver transplantation is not an option, high efficacy dialysis, such as daily hemodialysis, nocturnal hemodialysis or combination of hemodialysis and peritoneal dialysis is recommended. ${ }^{1}$ The gold standard treatment is pre-emptive sequential or combined liver-kidney transplantation. It should be planned when eGFR falls below $40 \mathrm{~mL} / \mathrm{min} / 1.73 \mathrm{~m}^{2}$ as after that the body oxalate burden increases rapidly. Isolated kidney transplantation is not recommended, because the liver is the only organ responsible for glyoxilate detoxification by the enzyme AGT and recurrence would eventually occur. Isolated liver transplantation is an option in selected patients, before established chronic renal failure. ${ }^{1,5}$ In the first case, the patient was initially proposed for sequential liver-kidney transplantation. Since renal function improved significantly and stabilized after liver transplantation, kidney transplantation was put on hold.

These case reports aim to raise awareness of the diagnosis of PH1 as an important underdiagnosed cause of ESRD in children. In Western Europe and North America it accounts for $1-2 \%$ of the pediatric ESRD population but this may reach $>10 \%$ in countries where there is a high rate of consanguinity, such as North Africa and Middle East. ${ }^{2,4}$ Its highly heterogeneous presentation and unfamiliarity with the disease among physicians (due to its rarity) play a major role in diagnostic delay. A high level of suspicion is necessary for an early diagnosis. ${ }^{2}$ An early and accurate diagnosis and treatment in $\mathrm{PH} 1$ are essential for achieving better short- and long-term outcomes. ${ }^{4}$

Disclosure of potential conflicts of interest: none declared

\section{References}

1. Cochat $\mathrm{P}$, et al; on behalf of OxalEurope. Primary hyperoxaluria Type 1 : indications for screening and guidance for diagnosis and treatment. Nephrol Dial Transplant 2012; 27: 1729-1736

2. Soliman NA, et al. Clinical spectrum of primary hyperoxaluria type 1: Experience of a tertiary center. Nephrol Ther 2017 May; 13(3): 176-182

3. Rhuma NR, et al. Mutational Analysis of AGXT Gene in Libyan Children with Primary Hyperoxaluria Type 1 at Tripoli Children Hospital. Saudi J Kidney Dis Transpl 2018; 29(1): 30-38

4. Isiyel E, et al. Molecular analysis of the AGXT gene in patients suspected with hyperoxaluria type 1 and three novel mutations from Turkey. Molecular Genetics and Metabolism 2016; 119: 311-316

5. Kurt-Sukur ED, et al. Primary hyperoxaluria type 1: a cause for infantile renal failure and massive nephrocalcinosis. Klin Padiatr 2015; 227: 293-295

6. Nair $\mathrm{P}$, et al. Combined liver and kidney transplantation in primary hyperoxaluria: a report of three cases and review of the literature. Saudi J Kidney Dis Transpl 2013; 24(5): 969-975

7. M'dimegh S, et al. Mutational Analysis of Agxt in Tunisian Population with Primary Hyperoxaluria Type 1. Annals of Human Genetics (2017) 81,1-10

8. Monico CG, Rossetti S, Olson JB, Milliner DS: Pyridoxine effect in type I primary hyperoxaluria is associated with the most common mutant allele. Kidney Int 2005;67(5):1704-1709

\section{Correspondence to:}

Inês Ganhão, MD

Serviço de Pediatria, Centro Hospitalar Barreiro-Montijo, Avenida Movimento das Forças Armadas, 2830-003 Barreiro

E-mail: inesieganhao@gmail.com 